\title{
Effect of Bagging on the Content of Sugar and Acid in Postharvest "Jinhuang" Mango Fruit
}

\author{
Changbin Wei, Jian Qiao, Xinming Tang, Qingze Yan, Lizhu Tang, Zhiling Ma* \\ Key laboratory of Tropical Fruit Biology, Ministry of Agriculture and rural areas, South Subtropical Crop Research Institute, Chinese \\ Academy of Tropical Agricultural Sciences, ZhanJiang, Guangzhou, 524091, China
}

\begin{abstract}
The glucose, fructose, sucrose, and organic acids in the pulp of "Jinhuang" mango were analyzed using the HPLC method and the effect of bagging on fruit quality was researched during the postharvest storage. The results showed that there was a certain difference in the content of sugar and acid among the three treatments. The effect of bagging treatments on fructose, glucose, and sucrose in the fruit of "Jinhuang" mango was mainly reflected in sucrose. The treatments of white bagging (WB) and black bagging (BB) delayed the production of sucrose and the decomposition of citric acid, and increased the content of ascorbic acid and quinine. Based on sugar-acid ratio, the flavor of the three treatments was evaluated as: $\mathrm{CK}>\mathrm{WB}>\mathrm{BB}$. The fruit quality of "Jinhuang" mango was affected by bagging treatments to a certain extent.
\end{abstract}

\section{Introduction}

Mango is a well-known tropical fruit, honored as the king of tropical fruits[1]. With the continuous expansion of global mango planting area and the year-to-year increase in output, mango has become one of the top five fruits in the world. By 2015, there were approximately 90 mango-growing countries in the world. The geographical area for mango cultivation is large, starting from the southern part of the Americas, to the south of Sichuan Province in China, spanning the area between $30^{\circ} \mathrm{N}$ to $30^{\circ} \mathrm{S}$. Asia is the region with the largest mango planting area and the highest yield, accounting for $85 \%$ of the world's total output; followed by the Americas, whose yield accounts for approximately $14 \%$ of the world's total. The country that has the largest mango harvest area in the world is India, with an area of more than 1 million hectares, accounting for $40 \%$ of the world's total; China ranks second globally, whose area of mango harvest accounts for $17 \%$ of world's total; Thailand has the third largest area of mango cultivation; Indonesia and the Philippines rank fourth and fifth; other major producing countries are Mexico, Egypt, Nigeria, and Bangladesh. Nowadays, in China and other countries, there are many researches on sugars and acids in fruits. Changes in sugar and acid content in fruits such as orange[2], pear[3], loquat[4], and cherry[5] have been studied.

As for studies on mango, the experimental results are not consistent owing to differences in cultivars and cultivation environments. In recent years, there have been considerable studies on fruit bagging. Bagging has a good efficacy in improving the appearance quality of fruits. However, research results on the effect of bagging on the intrinsic quality of fruits vary, especially when regarding its effect on sugars and acids in different mango cultivars. Studying the effect of bagging on the quality of mango can advance our understanding of the effect of cultivation measures on the improvement of fruit quality, which has great significance for improving the quality of mangoes and enhancing the international competitiveness of mangoes in China by optimizing the cultivation measures.

\section{Materials and methods}

\subsection{Experimental materials}

"Jinhuang" mango collected from Tiandong Mango Plantation in Baise, Guangxi were used for bagging experiments on April 5, 2017. Three treatment groups were set up: the control $\mathrm{CK}$, white bagging treatment (WB), and black bagging treatment (BB). In each treatment, three repetitions were conducted, and three trees per repetition were selected, and fruits with the same flowering period were selected for bagging treatments. Samples were collected on July 20, 2017, stored at room temperature of $26^{\circ} \mathrm{C}$, and subjected to tests every other day from 1 to 9 days after harvest.

\subsection{Experimental methods}

For analysis of glucose, fructose, and sucrose, as well as oxalate, malic acid, acetic acid, citric acid, ascorbic acid, and quinine, the methods in "Guide to the Experimental Analysis of Tropical Fruit Quality"[6] were referred to.

\footnotetext{
* Corresponding author: mbelle@163.com
} 


\section{Results and analysis}

\subsection{Effect of bagging on sugars in mango fruits}

\subsubsection{Equations of linear regression}

Mixed standard solutions and single standard solutions of fructose, glucose, and sucrose at a concentration range of $0.5-20 \mathrm{mg} / \mathrm{mL}$ were analyzed by high-performance liquid chromatography (HPLC). Correlation analysis was performed on the measured values of each type of sugars to obtain its linear regression equation. The correlation coefficient $\left(\mathrm{R}^{2}\right)$ of each equation was also calculated. As indicated in Table 1, the $\mathrm{R}^{2}$ values of linear regression equations of these three types of sugars were all above 0.999 .

Table 1. Linear regression equations and correlation coefficients of sugar measurements.

\begin{tabular}{|c|c|c|c|}
\hline $\begin{array}{c}\text { Measured } \\
\text { component }\end{array}$ & $\begin{array}{c}\text { Regression } \\
\text { equation }\end{array}$ & $\mathrm{R}^{2}$ & $\begin{array}{c}\text { Linearity } \\
\text { range } \\
(\mathrm{mg} / \mathrm{ml})\end{array}$ \\
\hline Fructose & $\begin{array}{c}\mathrm{Y}=81773.71 \mathrm{X}+ \\
4975.011\end{array}$ & 0.9999 & $0.5-20$ \\
\hline Glucose & $\begin{array}{c}\mathrm{Y}=57545.95 \mathrm{X}- \\
6663.779\end{array}$ & 0.9991 & $0.5-20$ \\
\hline Sucrose & $\begin{array}{c}\mathrm{Y}=87061.51 \mathrm{X}- \\
7429.614\end{array}$ & 0.9999 & $0.5-20$ \\
\hline
\end{tabular}

*In the regression equations, $\mathrm{Y}$ is the peak area, whereas $\mathrm{X}$ is the sugar concentration.

\subsubsection{Changes in sugar content for each treatment in postharvest stage}

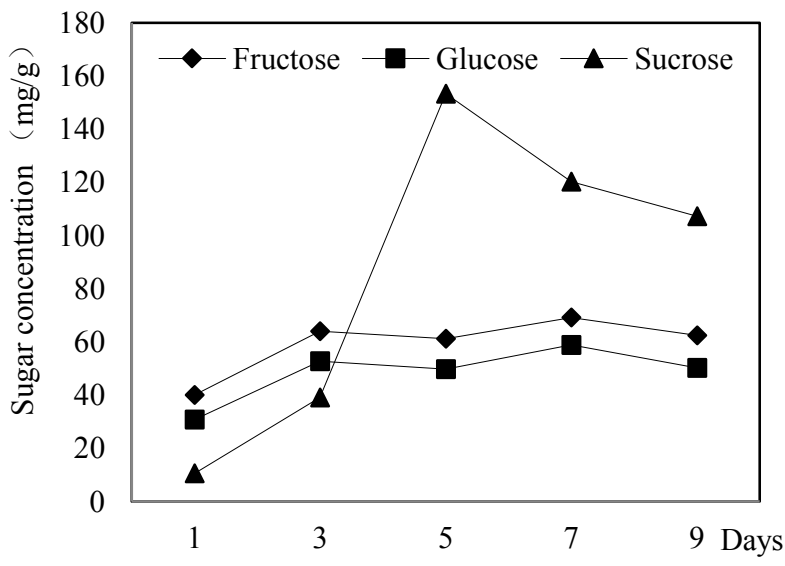

Fig. 1. Changes in the sugar content of CK during 1-9 days after harvest.

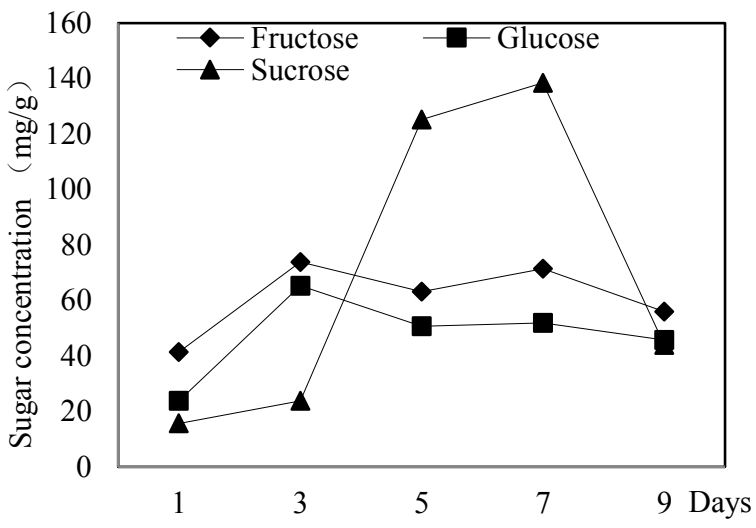

Fig. 2. Changes in the sugar content of WB during 1-9 days after harvest.

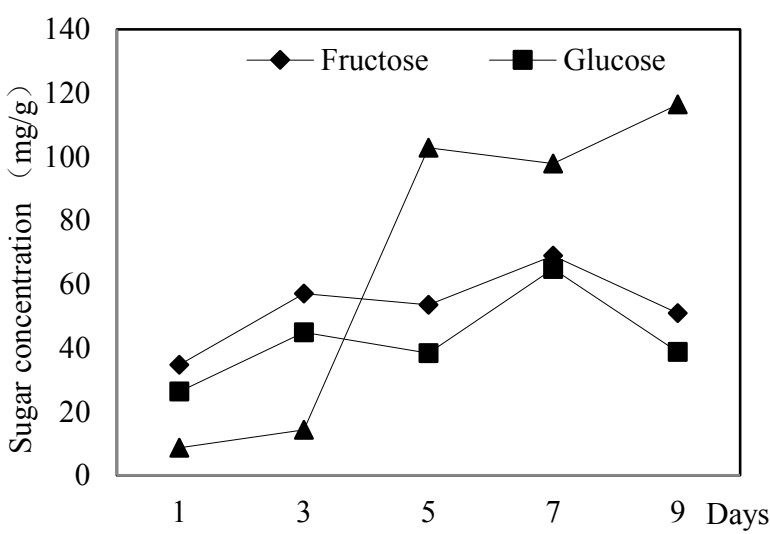

Fig. 3. Changes in the sugar content of BB during 1-9 days after harvest.

Fig. 1 to 3 show that fructose and glucose content in the pulp of the three treatment groups did not fluctuate significantly after harvest; the content of these sugars all increased slowly to a certain amount, then slowly decreased, and repeated the same fluctuation again, exhibiting a trend of slow increase followed by slow decrease. The fluctuation range of $\mathrm{CK}$ was smaller than that of the other two treatment groups; the postharvest fluctuation range of WB was larger in the earlier period and smaller in the later period; as for $\mathrm{BB}$, the fluctuation range was smaller in the earlier period and larger in the later period. On the first to fifth day after harvest, pulp sucrose content in the three treatments all slowly increased and then rapidly increased. However, on the fifth to ninth day after harvest, sucrose content was significantly different in each treatment: the sucrose content of CK reached its peak on the fifth day after harvest, and then decreased, but was still higher than its fructose and glucose content; the sucrose content of WB slowly increased until reaching its peak on the seventh day after harvest, and then decreased rapidly, but was lower than its fructose content in the end; as for BB, the sucrose content slowly decreased, then slowly increased, and reached its peak on the ninth day, but was higher than its fructose and glucose content in the end.

Therefore, it was indicated that the effect of bagging on fructose, glucose, and sucrose in "Jinhuang" mango fruits was mainly reflected in its effect on sucrose. 
Compared with $\mathrm{CK}, \mathrm{WB}$ and $\mathrm{BB}$ delayed the accumulation of sucrose, especially BB with a delay of four days. The effect of $\mathrm{WB}$ and $\mathrm{BB}$ on sucrose in mango was also reflected in the content level, as the peak sucrose content in BB was lower than that in the other two treatments.

\subsubsection{Differences of each type of sugars among the three treatments}

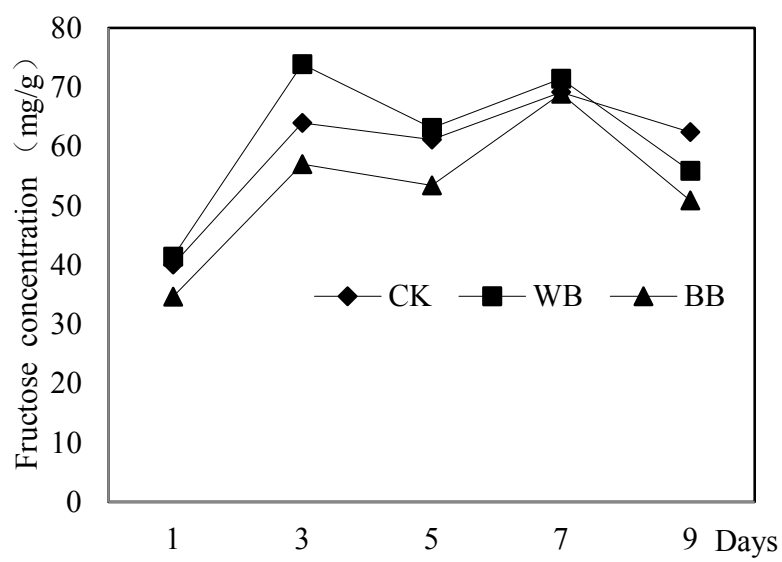

Fig. 4. Differences in fructose content among the three treatments during 1-9 days after harvest.

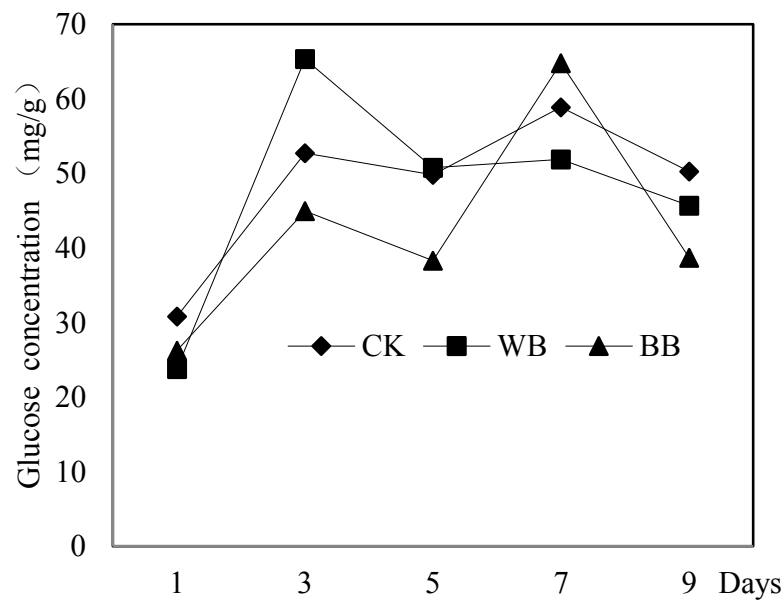

Fig. 5. Differences in glucose content among the three treatments during 1-9 days after harvest.

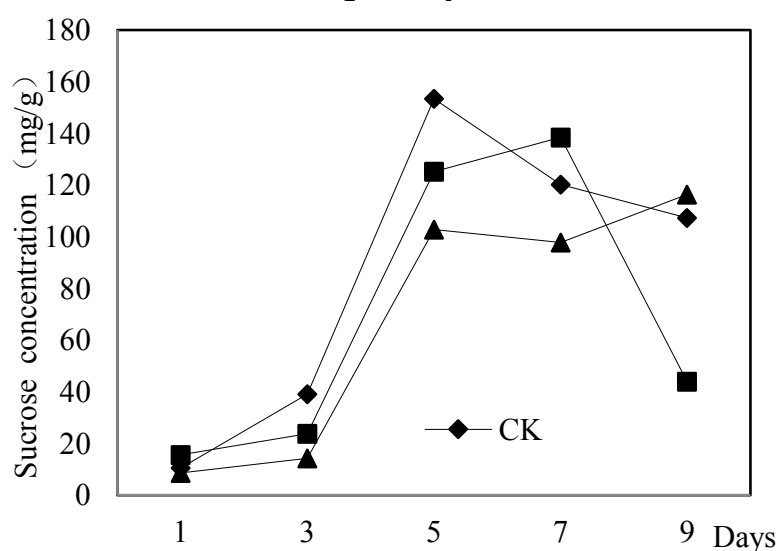

Fig. 6. Differences in sucrose content among the three treatments during 1-9 days after harvest.
Fig. 4 to 6 reveal that during 1-9 days after harvest, the content of the three sugar types in BB samples were all lower than that in the other two groups; the fructose content in WB samples was higher than that in $\mathrm{BB}$ for the entire testing time period and also higher than that in CK during 1-7 days after harvest. The fluctuation range of fructose content in each treatment was lower than that of glucose and sucrose content, and the fluctuation range of sucrose was the largest, which increased rapidly since the third day after harvest.

The above-mentioned analysis indicated that at the maturity stage of "Jinhuang" mango, WB facilitated the accumulation of fructose in fruits and increased fructose content in "Jinhuang" mango fruits; BB decreased fructose and glucose content and increased sucrose content in "Jinhuang" mango fruits; BB and WB both delayed the accumulation of sucrose in postharvest mangoes.

\subsection{Effect of bagging on organic acids in mango fruits}

\subsubsection{Linear regression equations}

Mixed standard solutions and single standard solutions of prepared organic acids were analyzed by HPLC. Correlation analysis was performed on the measured values of each type of organic acid to obtain its linear regression equation. The correlation coefficient $\left(\mathrm{R}^{2}\right)$ of each equation was also calculated. As shown in Table 2 , the $\mathrm{R}^{2}$ values of linear regression equations of all types of organic acids were above 0.999 . This indicated that the peak area of each sugar had a good linear correlation with its content under the set conditions of HPLC. Therefore, the organic acid content of the samples obtained by these linear regression equations had good accuracy.

Table 2. Linear regression equations and correlation coefficients of organic acid measurements.

\begin{tabular}{|c|c|c|}
\hline $\begin{array}{c}\text { Measured } \\
\text { component }\end{array}$ & $\mathrm{R}^{2}$ & $\begin{array}{c}\text { Correlation } \\
\text { coefficient }\end{array}$ \\
\hline Oxalate & $\mathrm{Y}=8216.075 \mathrm{X}+9440.86$ & 0.9995 \\
\hline Malic acid & $\mathrm{Y}=564.9531 \mathrm{X}+99.46098$ & 0.9999 \\
\hline Acetic acid & $\mathrm{Y}=357.1436 \mathrm{X}+291.5029$ & 0.9991 \\
\hline Citric acid & $\mathrm{Y}=810.1811 \mathrm{X}+268.8684$ & 0.9996 \\
\hline Ascorbic acid & $\mathrm{Y}=93952.06 \mathrm{X}+1603.903$ & 0.9999 \\
\hline Quinine & $\mathrm{Y}=93719.58 \mathrm{X}-40041.18$ & 0.9991 \\
\hline
\end{tabular}

*In the regression equations, $\mathrm{Y}$ stands for the peak area while $\mathrm{X}$ stands for the concentration of organic acid.

\subsubsection{Changes in content of organic acids for each treatment in postharvest stage}

Changes in the organic acid content of the three treatment groups during 1-9 days after harvest are illustrated in Figures 7 to 9. Changes in oxalate and 
malic acid content were all marginal in the three treatments with basically identical trends; the trends of acetic acid content fluctuations in the three treatments were also basically identical, all of which increased at first, then decreased, and again increased; the citric acid content of CK first continuously decreased and then increased, whereas the ascorbic acid and quinine content both continuously decreased throughout the entire testing period. In WB, however, citric acid content increased at first, then decreased, and again increased; ascorbic acid content increased at first, then decreased, and remained stable for a period before it decreased again; the fluctuation of quinine content appeared identical to that of citric acid content. As for BB, its citric acid content decreased at first and remained stable for a period before it decreased again, then it increased; its ascorbic acid content decreased at first and remained stable for a time before it decreased again; and its quinine content increased at first, then decreased, and again increased.

Overall, bagging or not and bagging types had minor effect on oxalate, malic acid, and acetic acid in "Jinhuang" mango fruits; however, they had more significant effect on the content levels of citric acid, ascorbic acid, and quinine as well as their fluctuations.

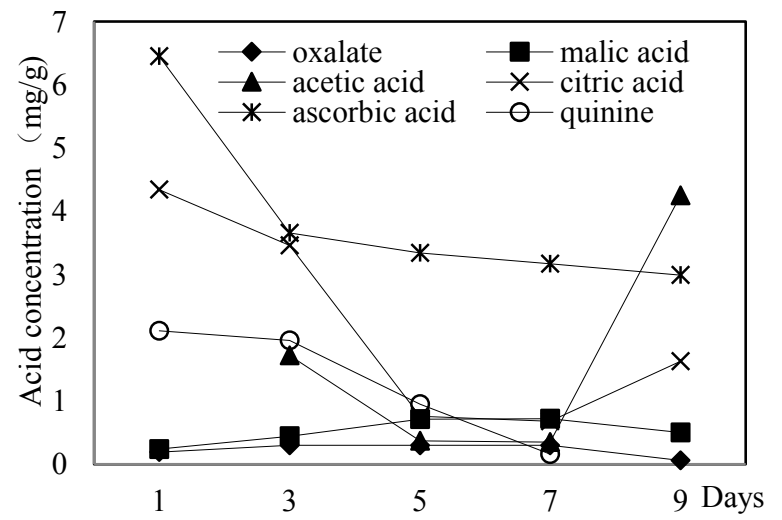

Fig.7. Changes in the organic acid content of CK during 1-9 days after harvest.

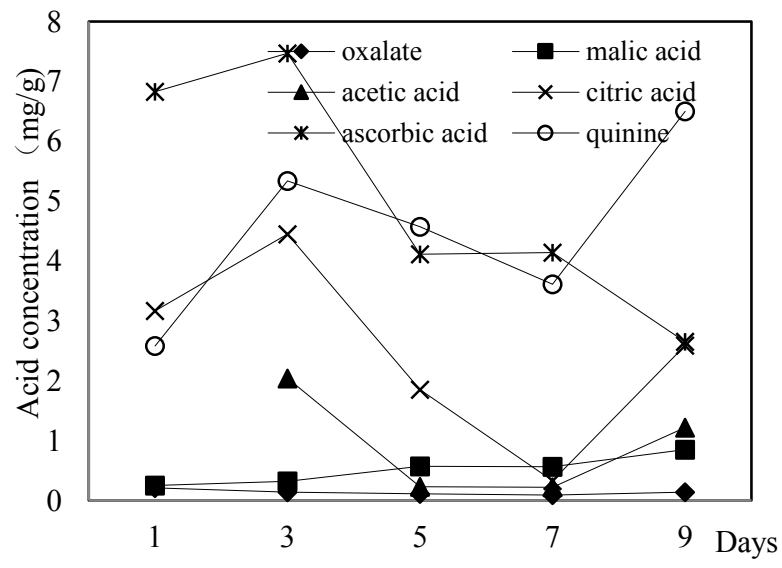

Fig.8. Changes in the organic acid content of WB during 1-9 days after harvest.

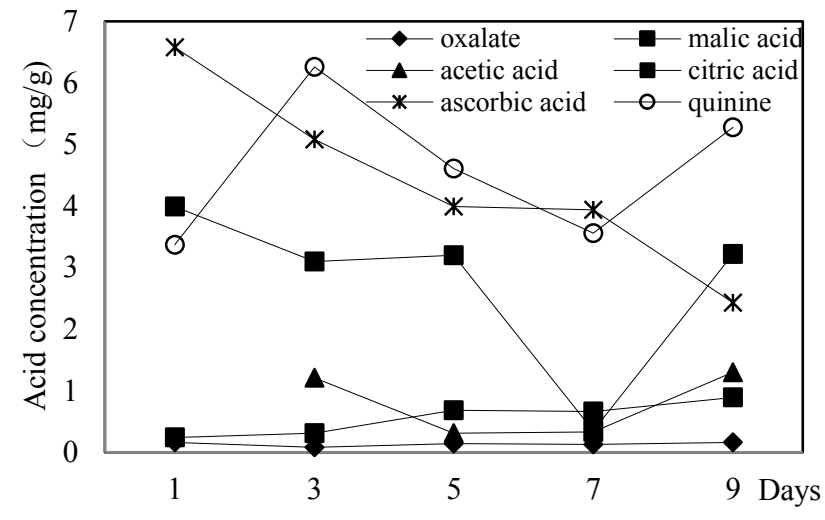

Fig.9. Changes in the organic acid content of BB during 1-9 days after harvest

\subsubsection{Differences of each type of organic acids among the three treatments}

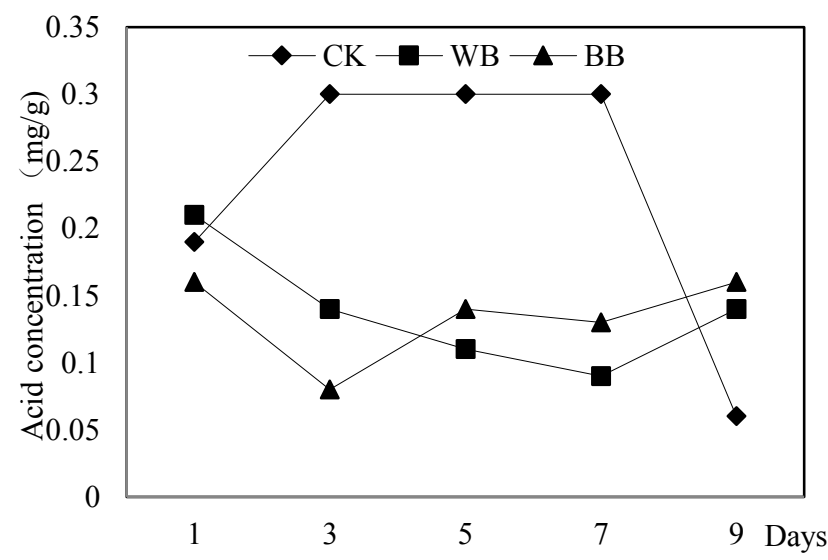

Fig.10. Differences in oxalate content among the three treatments during 1-9 days after harvest.

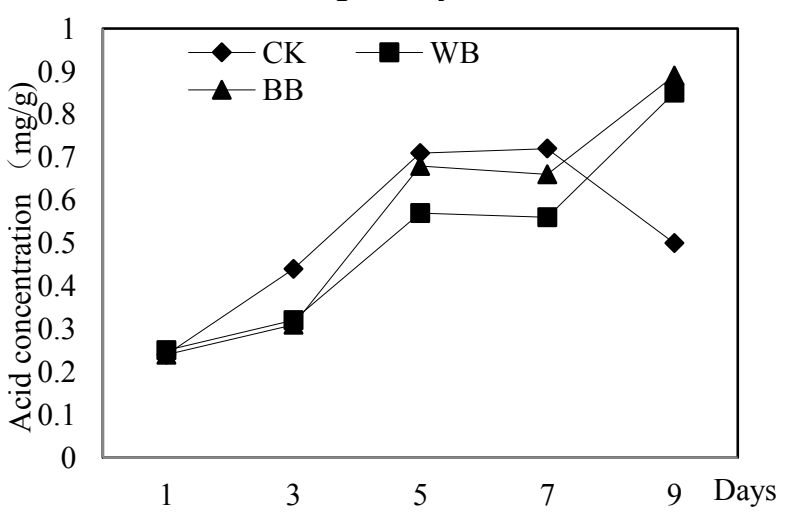

Fig.11. Differences in malic acid content among the three treatments during 1-9 days after harvest. 


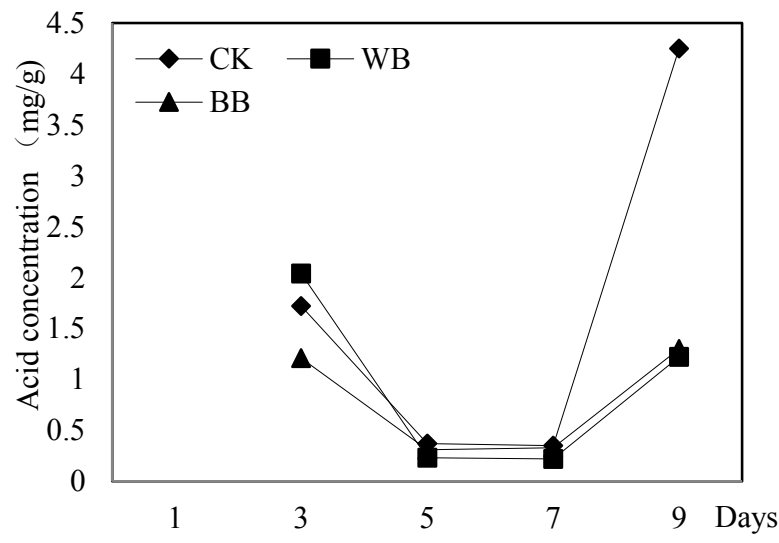

Fig.12. Differences in acetic acid content among the three treatments during 1-9 days after harvest.

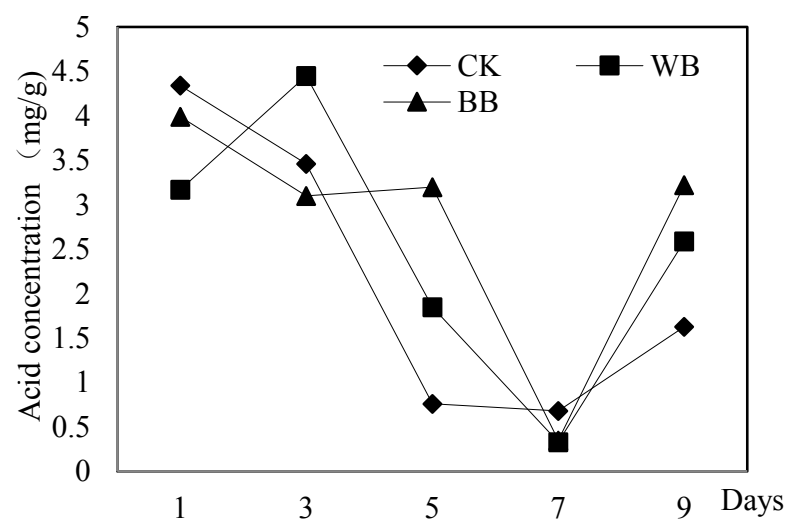

Fig.13. Differences in citric acid content among the three treatments during 1-9 days after harvest.

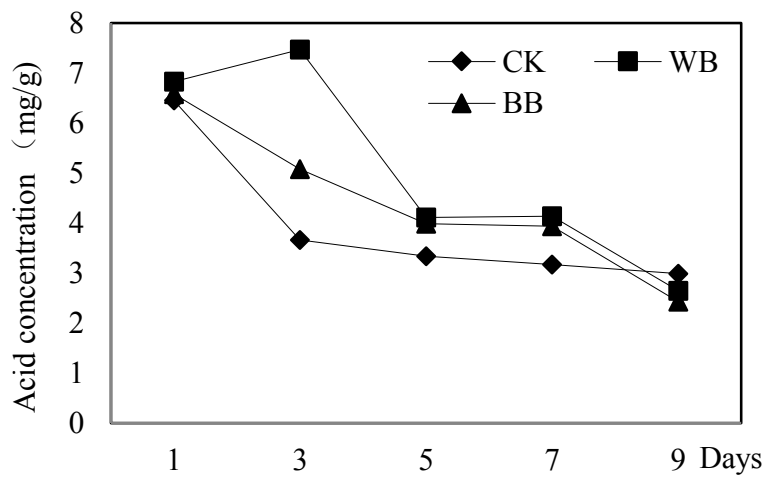

Fig.14. Differences in ascorbic acid content among the three treatments during 1-9 days after harvest.

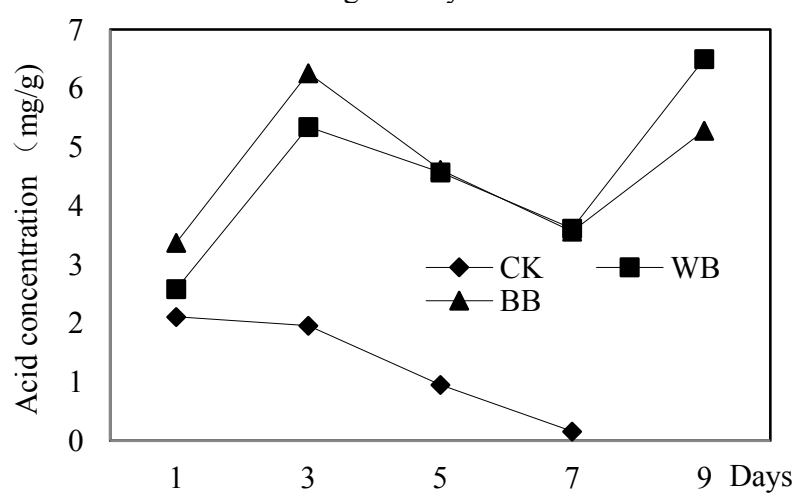

Fig.15. Differences in quinine content among the three treatments during 1-9 days after harvest.
As illustrated in the above Fig.10 to 15, ascorbic acid and quinine content in the bagging treatment was higher than that in $\mathrm{CK}$, whereas oxalate and malic acid content in CK was higher than that in the bagging treatment. The contents of oxalate, malic acid, acetic acid, ascorbic acid, and quinine content in $\mathrm{WB}$ and $\mathrm{BB}$ treatments were similar, whereas no acetic acid was detected on the first day. The difference in citric acid content was the most significant among the three treatments: $\mathrm{BB}$ and WB delayed the decomposition of citric acid, as the citric acid content of $\mathrm{BB}$ and $\mathrm{WB}$ reached the lowest value on the seventh day after harvest and then slightly increased; hence, citric acid content in the bagging treatment was higher than that in CK. As for quinine, both WB and BB treatments facilitated its accumulation and increased its content.

\subsection{Flavor comparison of "Jinhuang" mango}

Regarding fruit flavor, some scholars believed that it depends mainly on the total sugar content, total acid content, and sugar-acid ratio in fruits[7-9]. Therefore, in this study, we adopted the viewpoint of Liang Jun et al.[10] as a reference, in which the flavor of postharvest "Jinhuang" mango was evaluated based on the total sugar-acid ratio, as illustrated in Figure 16.

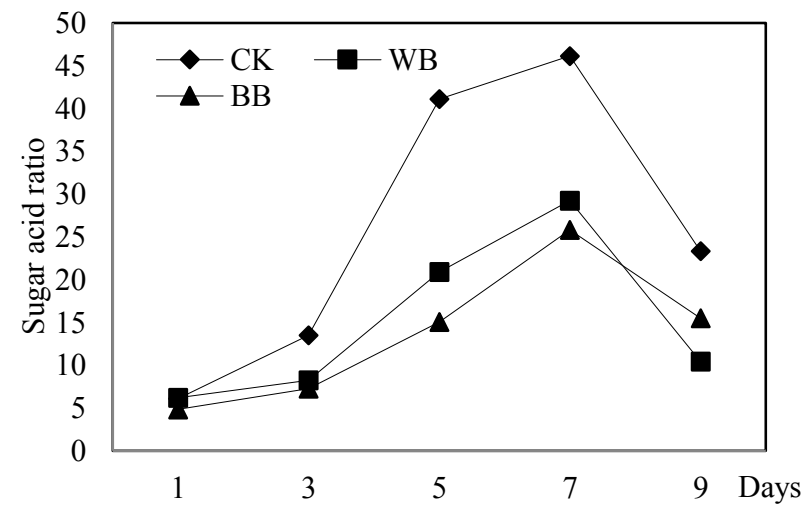

Fig.16. Changes in the total sugar-acid ratio of the three treatment groups after harvest.

The Fig. 16 revealed that the total sugar-acid ratios in all three treatment continuously increased during 1-7 days after harvest, indicating that the flavor of "Jinhuang" mango changed from sour to sweet and improved gradually; however, during 7-9 days after harvest, the total sugar-acid ratios in all three treatment groups decreased, indicating that the flavor of "Jinhuang" mango changed from sweet to sour and worsen. Moreover, the total sugar-acid ratio in $\mathrm{CK}$ was always higher than that in $\mathrm{WB}$ and $\mathrm{BB}$ during 1-9 days after harvest, indicating that the postharvest flavor of $\mathrm{CK}$ was better than that of $\mathrm{WB}$ and $\mathrm{BB}$; moreover, during 1-7 after harvest, the total sugar-acid ratio in WB was higher than that in $\mathrm{BB}$, indicating that the flavor of $\mathrm{WB}$ was better than that of $\mathrm{BB}$. In summary, the postharvest flavor in CK was better than in $\mathrm{WB}$ and $\mathrm{BB}$; the postharvest flavor in WB was slightly better than that in BB. 


\section{Conclusions}

Analysis of sugars and acids in "Jinhuang" mango under three treatments revealed that the effect of bagging on fructose, glucose, and sucrose in "Jinhuang" mango fruits was mainly reflected in sucrose. Compared with $\mathrm{CK}, \mathrm{WB}$ and $\mathrm{BB}$ delayed the production of sucrose, especially $\mathrm{BB}$ with a delay of four days. The effect of $\mathrm{WB}$ and $\mathrm{BB}$ on sucrose in mango was also reflected in the content level, as the peak sucrose content in BB was lower than that in the other two treatments. WB increased fructose content in "Jinhuang" mango fruits, whereas bagging treatments reduced glucose and sucrose content in "Jinhuang" mango fruits; the sugar content of $\mathrm{BB}$ was lower than that of the other treatments. Bagging and bagging types had minor effect on oxalate, malic acid, and acetic acid in "Jinhuang" mango fruits; however, they had more significant effect on citric acid, ascorbic acid, and quinine, as BB and WB delayed the decomposition of citric acid, and the citric acid content of $\mathrm{BB}$ and $\mathrm{WB}$ both reached their lowest value on the seventh day after harvest and then increased slightly; hence, citric acid content in the bagging treatment groups was higher than that in CK. Bagging treatments increased quinine and ascorbic acid content. Moreover, bagging treatments impaired the flavor of "Jinhuang" mango, as the postharvest flavor of CK was better than that of the bagging treatment, whereas the flavor of WB was slightly better than that of BB.

\section{Acknowledgments}

This study was supported by the grant from Central Public-interest Scientific Institution Basic Scientific Research Fund for Chinese Academy of Tropical Agricultural Sciences (No. 1630062016007; 1630062017017; 1630062017025); the Fund of Operation of Integrated Laboratory for SSCRI; the Fund of Operation of Key laboratory of Tropical Fruit Biology of Ministry of Agriculture and rural areas.

\section{References}

1. R.W. Li, G.D. Huang, M.H. Su, J.A. Zhou, Y.C. Chen, J. Sou. Agri., 44,875-878(2013)

2. L.Z. Liu, Hunan Agricultural University (Changsha, 2011)

3. H. Yuan, D.M. Tan, T. Li, S. Liu, X.S. Sun, D. Lei, A.D. Wang, Northern Fruits, 2, 14-16 (2017)

4. C.J. Deng, Q. Wu, Q.Z. Xu, J.M. Jiang, W.S. Hu, F. Jiang, X.P. Chen, S.Q. Zheng, Chin. J. Trop. Crops, 37,1747-1751 (2016)

5. G.Q. Wei, Y.G. Sun, Y. Sun, X.H. Yang, J. Fruit Sci., 31, 103-109 (2014)

6. C.B. Wei, Experiment guide for tropical fruit quality analysis (China Agricultural Publishing House,2018)

7. L. Jiang, H.H. Xu, F.F. Lin, Q.P. Qin, Chin. Fruits, 3, 26-29 (2012)
8. B.S. Wang, W.M. Gao, X.X. Ma, W. Yang, Hunan Agri. Sci., 18,50-53 (2014)

9. H.B. Wang, X.S. Chen, P.G. Xin, X.Y. Zhang, Z.J. Ci, J. Shi, H. Zhang, J. Fruit Sci., 24, 513-516 (2007)

10. J. Liang, Y. Guo, Y.L. Liu, M.M. Li, Z.Y. Zhao, J. Northwest A\&F University (Nat. Sci. Ed.), 39, 163170 (2011) 